\title{
Manual exploration and the perception of slipperiness
}

\author{
LAWRENCE E. M. GRIERSON and HEATHER CARNAHAN \\ University of Waterloo, Waterloo, Ontario, Canada
}

\begin{abstract}
In this article, we report on two experiments that examined the haptic perception of slipperiness. The first experiment aimed to determine whether the type of finger motion across a surface influenced the ability to accurately judge the frictional coefficient (or slipperiness) of that surface. Results showed that when using static contact, participants were not as good at distinguishing between various surfaces, compared with when their finger moved across the surface. This raises the issue of how humans are able to generate the appropriate forces in response to friction during grasping (which involves static finger contact). In a second study, participants lifted objects with surfaces of varying coefficients of friction. The participants were able to accurately perceive the slipperiness of the surfaces that were lifted; however, the grasping forces were not scaled appropriately for the friction. That is, there was a dissociation between haptic perception and motor output.
\end{abstract}

It has been proposed that human afferent visual input is separated into two fundamentally distinct pathways that allow for a division of labor in terms of how sensory information is processed (Milner \& Goodale, 1995). The first pathway, the dorsal stream, is responsible for the visual control of skilled action. Conversely, the ventral stream is important in the visual identification of objects: Transformations made in this stream allow for the creation of cognitive representations of the intrinsic and extrinsic characteristics of viewed objects (Milner \& Goodale, 1995). This dual-channel processing of visual input has been the topic of much research in the field of motor control; however, it is unclear whether or not a similar action-perception dissociation exists in other sensory modalities. Goodale and colleagues have provided both structural and behavioral evidence that the haptic system shares properties of the visual system, in terms of separate action-perception pathways (James, Humphrey, Gati, Servos, Menon, \& Goodale, 2002; Westwood \& Goodale, 2003). More recently Prather, Votaw, and Sathian (2004) have confirmed the notion of separate dorsal and ventral pathways for the processing of haptic input, depending on whether this input is to be used for action or perception.

There is other evidence that supports the notion of a haptic perception-action dissociation. The size-weight illusion is a robust phenomenon discovered over a century ago, in which two objects of various sizes are manipulated so that they have identical masses. When individuals are asked to lift these objects while using their sense of vision,

This project was supported by a research grant awarded to H.C. from the Natural Science and Engineering Research Council of Canada. Correspondence concerning this article should be addressed to $\mathrm{H}$. Carnahan, Department of Surgery, The Wilson Centre, 200 Elizabeth Street, 1 ES 559, Toronto, ON, M5G 2CH Canada (e-mail: heather.carnahan@ gmail.com). they consistently report that the small object feels heavier than the large object (Charpentier, 1891). However, Gordon and his group have shown that grasping forces used when lifting a large box are greater than those used when lifting a smaller box, even though individuals perceive the small box to be heavier than the large box, thus eliciting a dissociation between the action and perception components of the task (Gordon, Forssberg, Johansson, \& Westling, 1991a, 1991b). In a similar study, Ellis and Lederman (1993) showed that haptic input alone was capable of producing this illusory effect to a greater degree than a solely visual condition. When lifting objects in a congruent manner that vary in both size and mass, larger objects are perceived as heavier, and more force is used to grasp them (Johansson \& Westling, 1990). With the size-weight illusion, the heavier objects are perceived as being lighter, even though more force is used to grasp them. These data could be interpreted to suggest that there is a dissociation between the haptic perception of mass, and the action or motor output required to lift a particular mass.

The relationship between size and weight has been extended to show that the effects of object mass and size on weight perception follow from invariants of rotational dynamics. That is, weight perception depends on invariances of the stimulus, such as an object's rotational inertia (Amazeen \& Turvey, 1996). This notion has recently been extended to highlight the role of object mass, volume, and symmetry of the inertial characteristics of the object, in the perception of heaviness (Shockley, Carello, \& Turvey, 2004).

Friction perception has also been an aspect of haptic information processing that contributes to subjective sensations. Coefficient of friction between grasp surfaces and the fingers has been shown to be both necessary and important in the scaling of grasp forces; this variable needs to be processed via the haptic perceptual system in order for a lift and hold to be conducted appropriately. Johans- 
son and Westling (1984) and Cadoret and Smith (1996) have shown that increases in grip forces are proportionately scaled to the inverses of the coefficients of friction, independent of variations in surface texture.

The perception of skin-to-surface coefficient of friction has also been examined in terms of an individual's ability to create subjective sensations using digital exploration. Johnson, Hsiao, and Yoshioka (2002) have examined neural coding as it relates to the perception of texture, and they showed linearity between subjective experience and the neural activity on which it is based. Thus, one would expect a strong relationship between the subjective experience or perception and the activity that created the neural activity (motor action). Smith and Scott (1996) explored the psychophysical relationship between actual and perceived friction on flat, featureless surfaces, and showed significant correlations between the subjective ratings of friction and the measured coefficients of friction. They also showed that during exploration, participants maintained a constant normal force while varying the degree of tangential force applied, suggesting that the finger contains tangential-force-sensitive receptors that are important in the perception of smooth-surface coefficients of friction (Pare, Carnahan, \& Smith, 2002; Smith \& Scott, 1996). Johnson and colleagues (Blake, Hsiao, \& Johnson, 1997; Johnson, 2001) have suggested that specific receptors in the finger pads (rapidly adapting Meissner afferents) are sensitive to slip and motion across the skin. Lederman and Taylor (1972) conducted a study on individuals' ability to subjectively perceive surfaces with varying textures and friction coefficients during static contact. The results of this study indicated that individuals were able to detect differences in surface texture but not in coefficient of friction, strengthening the proposal that for friction to be perceived, dynamic or tangential motion across the surface is necessary. This conclusion thus raises the issue of how prehensile forces are properly scaled to the coefficient of friction of grasping surfaces. If dynamic exploration of surfaces is necessary for the accurate perception of friction, is it also necessary for scaling of forces when lifting objects? Cadoret and Smith (1996) have shown that it is not necessary. The purpose of the following experiments was to systematically investigate this issue.

\section{EXPERIMENT 1}

Experiment 1 aimed to determine how exploratory conditions influenced the subjective rating of coefficient of friction. That is, the study was designed to examine the ability to judge coefficients of friction in both static and dynamic exploration conditions. Whereas it would be expected that tangential finger motion is necessary for the perception of slipperiness, it is necessary to empirically determine this, particularly in light of the observation that scaling of finger forces to friction takes place during grasping and lifting, when the digits remain relatively stable on an object (Cadoret \& Smith, 1996). It is hypothesized that coefficient of friction will be difficult to judge when the finger is static; however, when there is dynamic, tangential motion of the finger across the surface, judgments of friction will be more accurate.

\section{Method}

Participants. The participants in this study were $20(5$ male and 15 female; mean age $=23.2$ years) students from the University of Waterloo. All of the volunteers provided informed consent, and the project was approved by the University of Waterloo Office for Research Ethics.

Apparatus. The apparatus for the perceptual phase of Experiment 1 consisted of a curtained screen that visually separated the participants from a horizontal tabletop where one of five surfaces was presented. The surfaces-UHMW polyethylene, PVC plastic, glass, rosin-coated glass, and lubricated polyethylene-were all macroscopically flat and featureless, with varying coefficients of friction. The surfaces were chosen based on the findings from a pilot study that showed differences in skin-to-surface coefficients of friction between the three untreated surfaces, and the rosin and lubricant treatments were added to the surfaces to extend the range of the coefficients of friction. In the second phase of Experiment 1, the coefficients of friction of each surface, for each participant, were determined. A six-axis force transducer (Nano F/T transducer, ATI Industrial Automation, Garner, NC) was fitted with removable caps (35-mm diameter, $0.4-\mathrm{mm}$ thick) made out of the five previously mentioned surfaces. The total mass of the apparatus was $300 \mathrm{~g}$. These caps served as the grasping surface for the performance of slip trials, which were used to determine each participant's skin-tosurface coefficient of friction. Force data were sampled at $200 \mathrm{~Hz}$ and filtered at $10 \mathrm{~Hz}$.

Procedure. All of the participants washed and dried their hands before the beginning of the testing session. In the first phase of the experiment, each of the five surfaces was presented to the participants in one of six different exploratory conditions. The six conditions were: static finger contact, finger rolling, finger tapping, leftright finger translation, up-down finger translation, and free finger exploration. In the static condition, the participants were asked to reach through the curtain and place and hold the pad of their index finger on the presented surface. The finger-rolling exploratory procedure required the participants to make contact with the surface in a manner similar to the way contact was made in the static condition; however, upon making contact, the individual was to pivot his or her finger around a central pressure point as one would when having fingerprints taken. The finger-tapping condition required that the participant repeatedly tap his or her index finger on the presented surface. The left-right and up-down translation conditions allowed the participants to slide their fingers along the surface presented in either back-and-forth or up-and-down motions, respectively. The free-exploration condition allowed the participants to examine the presented surface any way they chose. It was reasonable to restrict the exploration to a single finger, because Symmons and Richardson (2000) have shown that, if given the opportunity to explore surfaces with any number of fingers, participants spontaneously use only one finger. Following the completion of each trial, each participant was required to give a subjective estimate of the surface's slipperiness by marking a point on a visual analogue scale. This scale was consistent with the scale used by Smith and Scott (1996), and consisted of an $18-\mathrm{cm}$ line with one end marked "least slippery" and the other end marked "most slippery." Each surface was randomly presented and was rated four times for each of the blocked exploratory conditions. The order in which each exploratory condition was presented was randomized across participants. After lifting either the rosin-coated or the lubricated surface, participants washed and dried their hands. To ensure that the hand washing itself did not influence either perception or action, we conducted pilot work in which the three intermediate conditions were performed in a block alone (with no hand 
washing), and then in a block that included both the rosin-coated and the lubricated surfaces, in which hand washing took place after each trial. The performances on the intermediate conditions were compared across the two phases of the study, and no differences were found $(p>.05)$. In addition, during this pilot work, all of the participants subjectively reported that all of the surfaces were equally smooth.

For the second phase of the experiment (the coefficient of friction determination phase), the participants lifted the transducer using their index finger and thumb. The participants were then instructed to slowly loosen their grip on the transducer until it began to slip through their fingers. This procedure was repeated three times for each surface. The load (tangential) and grip (normal) force values were determined at the moment of the onset of the slip. The load force was divided by the grip force for each of three trials per surface, and these values were then averaged to produce mean coefficients of friction for each surface (Cadoret \& Smith, 1996). The possibility exists that the participants were able to use auditory cues to provide information about the surfaces being explored; however, it was the experimenters' assessment that obvious auditory cues were not produced.

Analyses. Estimated ratings of the perceived coefficient of friction for each participant were determined by measuring the distance he or she marked along the visual analogue scale. These ratings were then correlated with the actual coefficients of friction for each participant, as determined by the slip trials in the second phase of the study. Also, the mean (average of four trials) perceptual estimates were analyzed in a 6 (condition: static, rolling, tapping, left-right translation, up-down translation, free exploration) $\times 5$ (surface: UHMW polyethylene, PVC plastic, glass, rosin-coated glass, lubricated polyethylene) ANOVA.

A one-way five-factor (surface: UHMW polyethylene, PVC plastic, glass, rosin-coated glass, lubricated polyethylene) ANOVA was run on the coefficients of friction that were determined in the second phase of the experiment. Effects significant at $p<.05$ were further analyzed using the Tukey HSD method for post hoc comparison of means.

\section{Results and Discussion}

Coefficient of friction determination phase. There was a main effect of surface $[F(4,56)=4.29, p<.05]$. Three groupings of coefficients of friction emerged, with the rosin- and lube-coated surfaces the two extremes and the three remaining surfaces (glass, PVC, UHMW) appearing similar and intermediate to the other two surfaces. However, there were statistically significant differences only between the rosin-coated glass and the other four surfaces (see Figure 1).

Perceptual phase. The ANOVA conducted on the mean perceptual estimates produced an interaction between the exploratory procedure used and the surface being explored $[F(20,380)=4.65, p<.05]$. As seen in Figure 2, in the static finger condition, the participants were able to distinguish between the two extreme surfaces (rosin-coated glass and lubricated polyethylene). However, they were not able to distinguish between the three surfaces with intermediate coefficients of friction (glass, PVC plastic, and UHMW polyethylene), or distinguish between the intermediate surfaces and the two extreme surfaces. For the five remaining exploratory conditions that allowed for finger movement, the participants were able to distinguish between the intermediate surfaces and the extreme surfaces.

When the subjective estimates of perceived friction were compared with the coefficients of friction for each of the presented surfaces, significant correlation coefficients were obtained for the exploratory procedures that involved tangential motion: For all six surfaces, the mean correlation for the free exploration condition was $r=$ $-.206(p<.05)$, for the left-right translation condition it was $r=-.199(p<.05)$, and for the up-down translation condition, the value was $r=-.194(p<.05)$. Conversely, the static $(r=-.039, p>.05)$, rolling $(r=-.076$, $p>.05)$, and tapping $(r=-.094, p>.05)$ conditions did not show significant correlations between the ability to rate friction and the actual coefficient of friction.

In summary, the coefficient of friction differences between the surfaces were more accurately perceived during the exploratory procedures that involved tangential

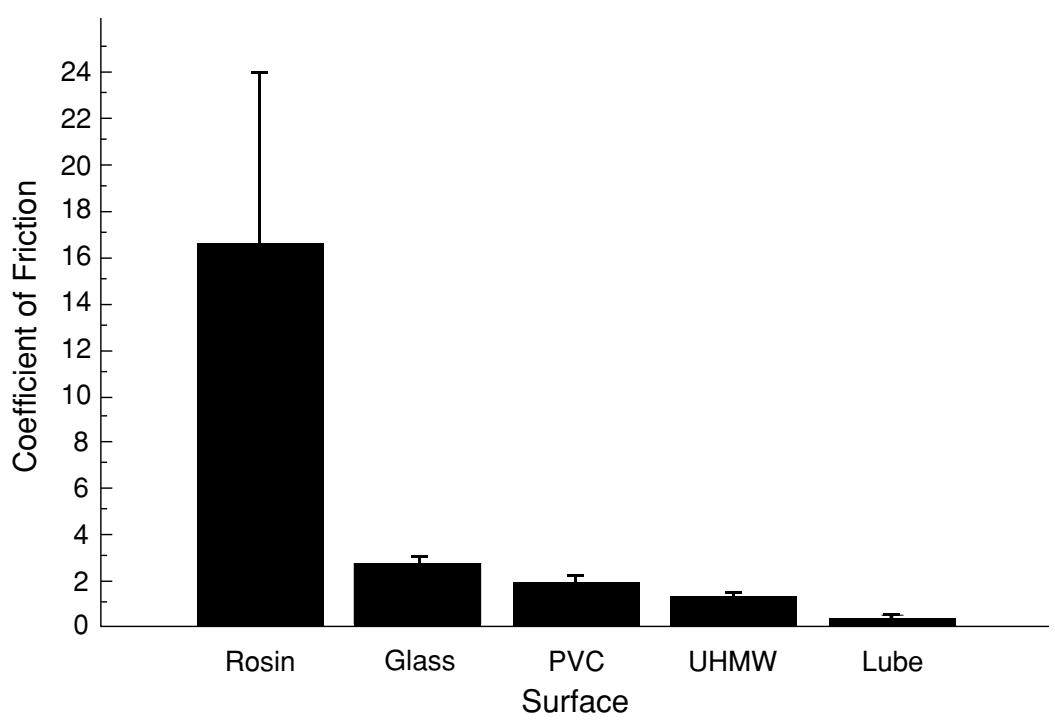

Figure 1. Coefficients of friction for each surface in Experiment 1. 


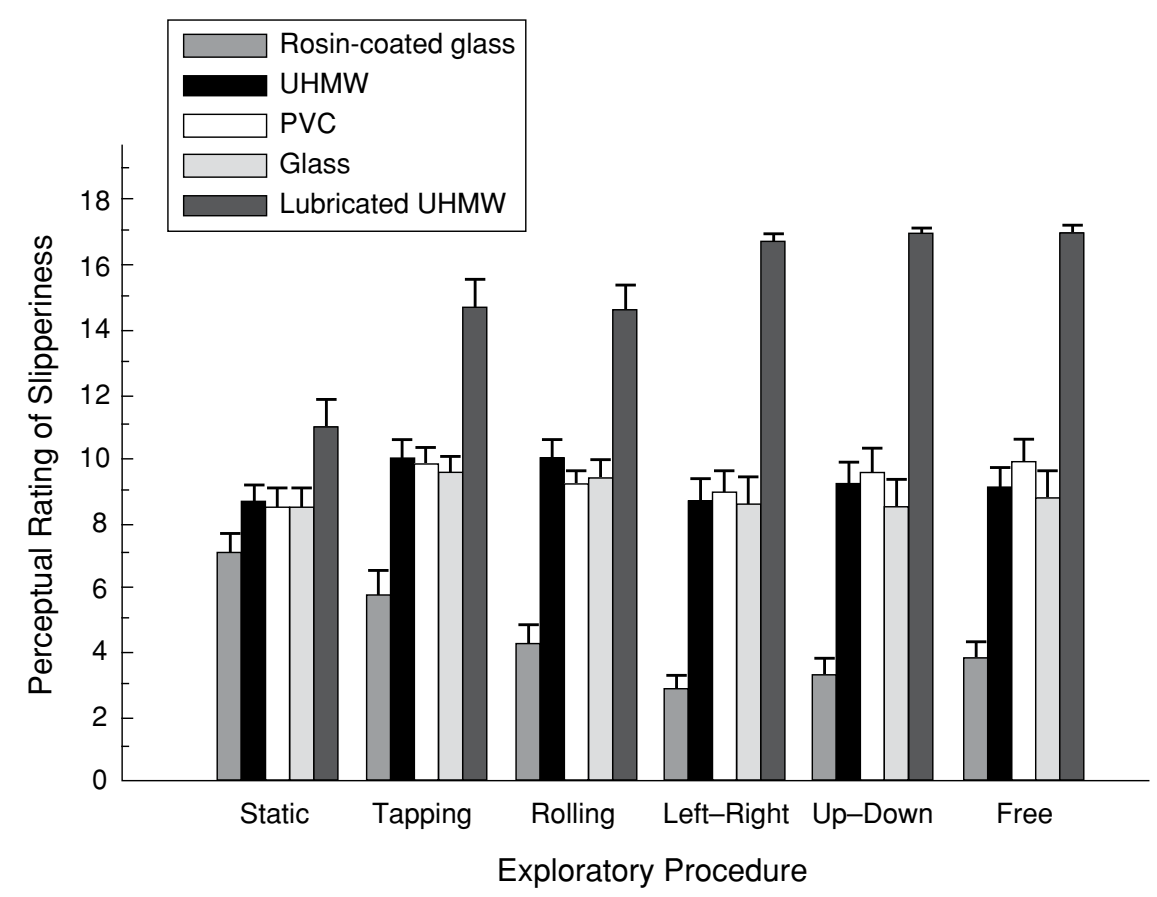

Figure 2. Perceptual rating of slipperiness as a function of exploratory procedure for Experiment 1.

motion (left-right translation, up-down translation, and free exploration). The correlation analysis supports the interaction found between the exploratory procedure and the explored surface, by showing direct relationships between the surfaces' actual coefficients of friction and the participants' ability to appropriately rate coefficient of friction when using tangential motion but not static contact. These findings are consistent with the studies (Lederman \& Taylor, 1972; Smith \& Scott, 1996) that show that tangential motion is necessary for the accurate perception of surface friction.

\section{EXPERIMENT 2}

The results of Experiment 1 showed that the participants were able to identify differences in the slipperiness of the surfaces they were exploring in all conditions except the static exploratory procedure. However, prehension requires finger contact with no tangential motion on the grasping surface (Cadoret \& Smith, 1996; Johansson \& Westling, 1984). Thus, it seems that there may be differences between how friction is evaluated when modulating grasping forces, and how it is evaluated when making perceptual judgments about slipperiness. Experiment 2 examined whether the pinching action that is used for grasping allows for accurate perceptual judgments regarding slipperiness. We examined the force output when participants lifted objects with grasping surfaces that had different coefficients of friction, and compared this with their ability to judge the coefficients of friction of these surfaces. It was expected that grip forces would be scaled according to surface friction, but on the basis of the findings from Experiment 1 that showed that tangential motion was required to perceive slipperiness, the participants would not be able to accurately judge the slipperiness of the object being lifted. This pattern of findings would be consistent with the notion of an action-perception dissociation for the haptic system. On the basis of the first study, there are three ranges of stimuli: the most slippery (with lubricant), the least slippery (with rosin), and the three surfaces with an intermediate slipperiness that did not differ from each other (glass, PVC, UHMW). It was expected that there would be variations in performance across the range of coefficients of friction, and there would be consistency of performance when the three surfaces with similar coefficients of friction were compared.

\section{Method}

Participants. Twenty students from the University of Waterloo ( 11 males and 9 females; mean age $=20.5$ ) participated in this study. None of the participants had been involved in the first experiment. All of the volunteers provided informed consent, and the project was approved by the University of Waterloo Office for Research Ethics.

Apparatus. The apparatus for Experiment 2 included the same six-axis force transducer with the adjustable caps composed of lubricated UHMW polyethylene, UHMW polyethylene, PVC plastic, glass, and rosin-coated glass that were used in Experiment 1 $($ mass $=300 \mathrm{~g})$. Also, the same $18-\mathrm{cm}$ visual analogue scale that was used in Experiment 1 was used in the present study to obtain the subjective perceptual ratings.

Procedure. The experiment was conducted in two phases. The first part, the motor phase, required the participants to lift the transducer with varying grasping surfaces approximately $10 \mathrm{~cm}$ above the laboratory table. Each trial was conducted with no vision and, 
upon completing the lift, the participants were asked to subjectively rate their perceived level of slipperiness for each surface. The second part of the experiment involved slip trials that were used to determine the actual coefficient of friction for each individual, for each surface. All of the participants washed and dried their hands before beginning the testing session.

During the motor phase of this experiment, the participants were seated at a table with a curtain separating them from the force transducer. The participant reached through the curtain and, with the aid of the experimenter, located the transducer, so that they could grasp and lift the transducer using the index finger and thumb of their dominant hand. The experimenter would receive the participant's hand and position it so that the aperture of the thumb and index finger was appropriate for the force transducer to be lifted. The transducer was then placed in between the participant's finger and thumb, and the lift was initiated. Before returning the transducer to the table, the participant provided a subjective estimate of the surface's slipperiness, using his or her free hand, by marking a point on the $18-\mathrm{cm}$ visual analogue scale. The force transducer measured and recorded the grip and load forces, and torques, as well as the grip force and load force rates. Each grasping surface was presented, lifted, and rated 10 times, in random order. Hands were washed after every trial.

In the second part (coefficient of friction determination phase) of Experiment 2, participants performed slip trials. Slip trials were completed in exactly the same way they were in the coefficient of friction determination phase of Experiment 1.

Analysis. The transducer measured grip forces - the forces along the grip axis defined by joining the centers of the two grasping surfaces. Additionally, load forces, defined as the vector sum of the two perpendicular forces acting in the orthogonal plane to the grip force axis, were measured. Forces across the two digits (index finger and thumb) were grouped, because the object was symmetrical and the constraints on each digit were identical. Resultant torque, the rates of grip and load force production, and the timing of the peaks of the generated forces and their rates were also calculated. The timing of the peaks was based on the occurrence of the respective peaks relative to initial object contact, with object contact defined as the point at which grip force deviated more than one standard deviation above the baseline. Each of these dependent measures was analyzed in a 10 (trial) $\times 5$ (surface: UHMW polyethylene, PVC plastic, glass, rosincoated glass, lubricated polyethylene) repeated measures ANOVA. Ef- fects significant at $p<.05$ were further analyzed using a Tukey HSD post hoc method for the comparison of means. Estimated ratings of the perceived coefficients of friction and the actual coefficients of friction were also correlated with the motor output values (grip force, rate of grip force generation, and torque) collected from the force transducer. These values were compared in order to identify any similarities or differences between the perceptual and motor outputs.

\section{Results and Discussion}

Coefficient of friction determination phase. The analysis of coefficient of friction showed significant differences between the lubricated polyethylene and the rosin-coated glass, and the rosin-coated glass and the glass surface $[F(4,76)=3.69, p<.05]$ (see Figure 3 ). Similar to the results of Experiment 1, the coefficients of friction for the three intermediate surfaces did not differ (PVC, UHMW, glass). The order and the values of the intermediate surfaces' coefficients of friction differed between the first and the second experiments. This difference was probably due to the measurement being dependent on the characteristics of the participants' skin.

Perceptual estimates. The analysis of the perceptual estimates produced a main effect of surface $[F(4,76)=$ $37.8, p<.01]$. An interaction between trial and surface was also found $[F(36,684)=3.05, p<.01]$. The ratings for UHMW and rosin remained stable across trials, whereas the ratings for PVC and glass increased (felt more slippery) with practice (the rating for PVC Trial 1 was higher than the ratings for Trials 6 and 8; the rating for glass Trial 1 was higher than the ratings for Trials 8 and 10). On the contrary, ratings for lubricated glass decreased (it was perceived as less slippery) with practice (the rating for Trial 1 was higher than the rating for Trial 10). The rosin-coated glass was perceived as the least slippery surface and the lubricated polyethylene as the most slippery (see Figure 4). There were no perceived differences in the

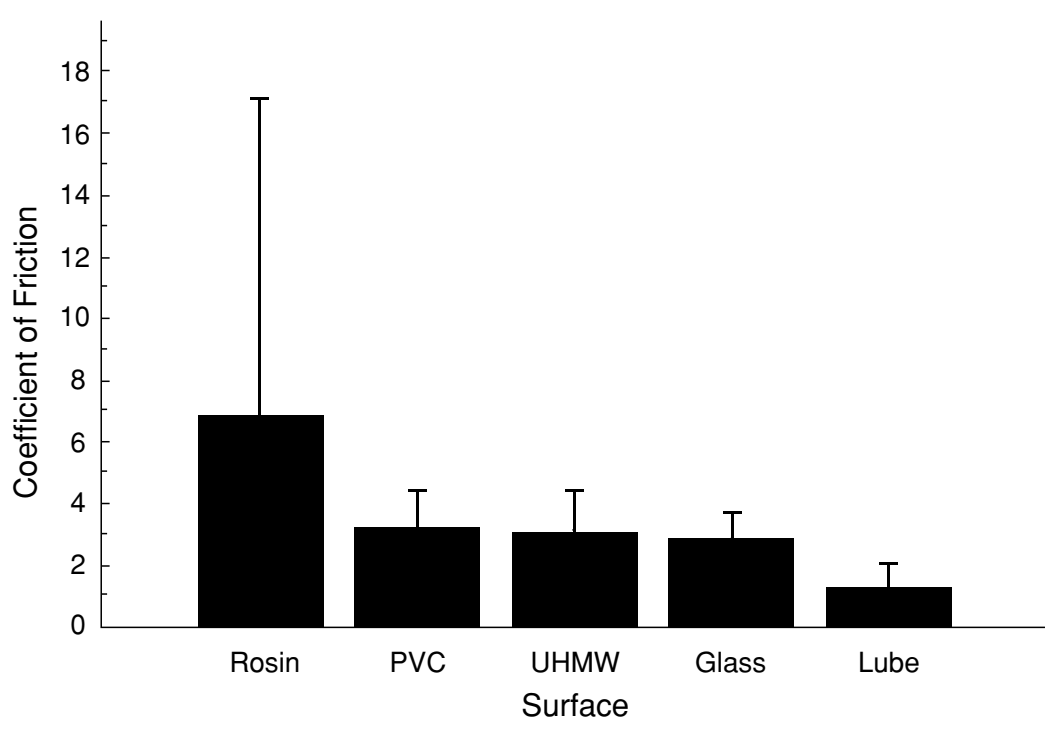

Figure 3. Coefficients of friction for each surface in Experiment 2. 


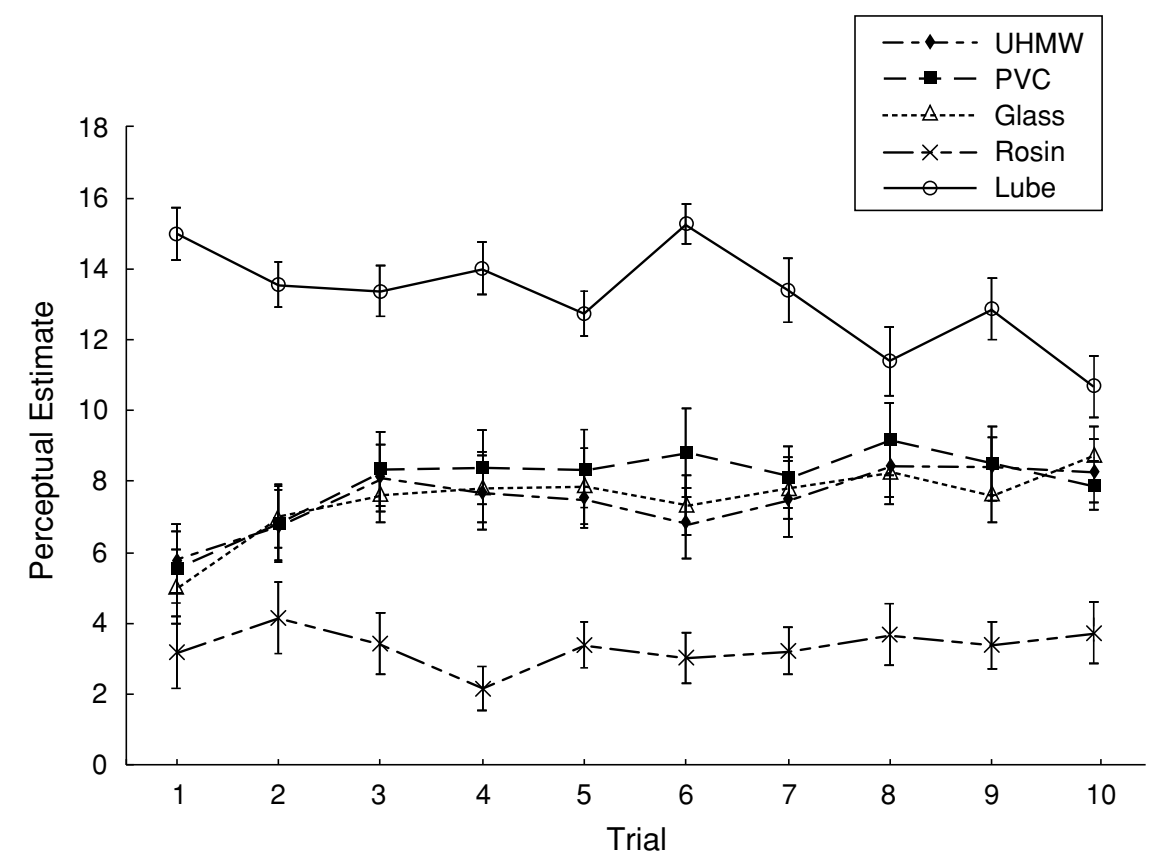

Figure 4. Perceptual estimates, plotted as a function of trial for each of the surfaces in Experiment 2.

slipperiness of the three intermediate surfaces (PVC plastic, UHMW polyethylene, and glass).

Peak grip force. The analysis of peak grip force produced main effects of both surface $[F(4,76)=59.17, p<$ $.01]$ and trial $[F(9,171)=14.8, p<.01]$, as well as an interaction of these two factors $[F(36,684)=1.78, p<.01]$.
Typical grip force curves are presented in Figure 5. Grip force did not differ statistically across trials for the UHMW and rosin surfaces. However, for the remaining three surfaces, grip force decreased (PVC Trial 1 force was greater than the force for Trials 2 and 5; glass Trial 1 force was greater than the force for Trial 7; lubricated glass Trial 1
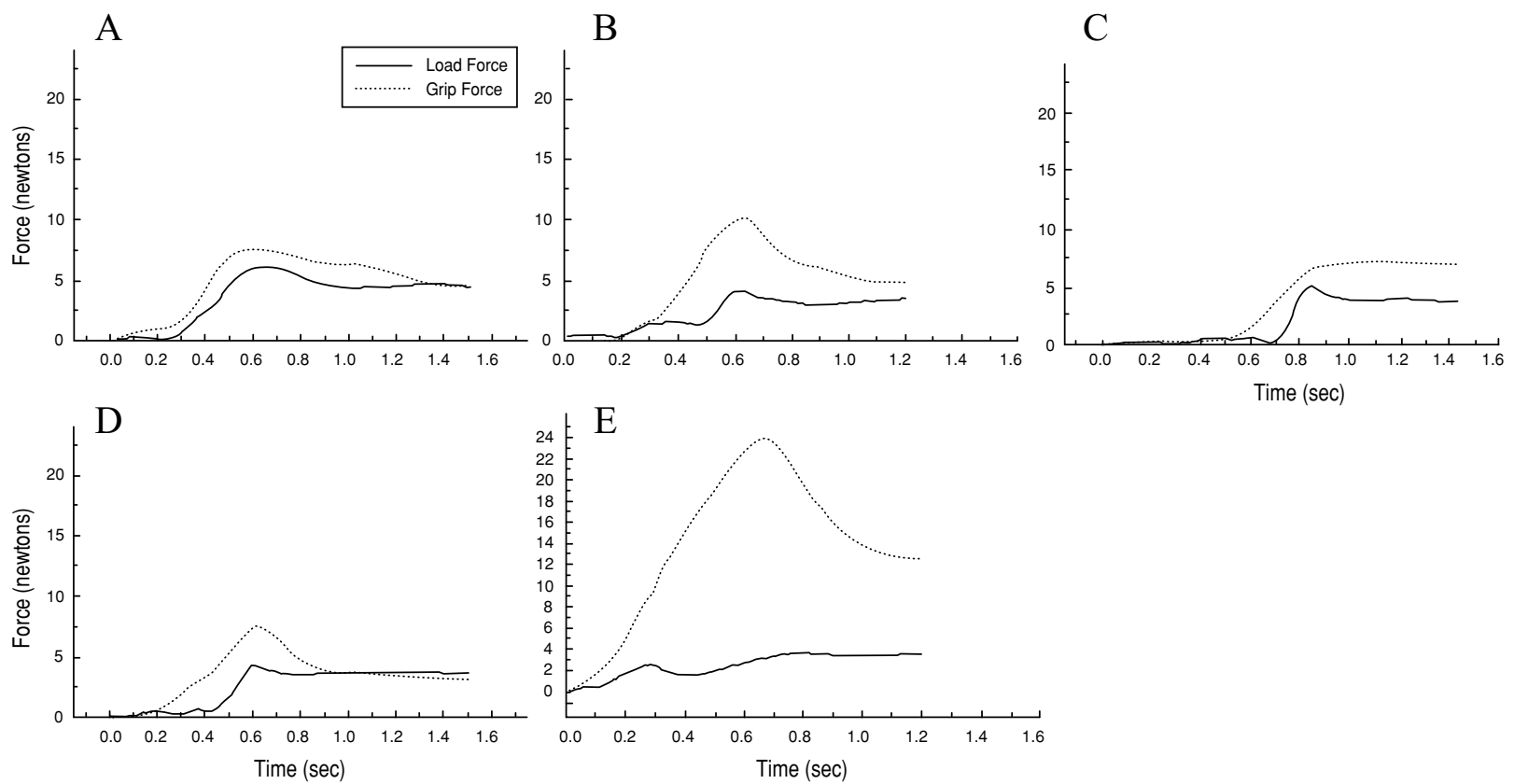

Figure 5. Typical force profiles for lifts from 1 participant, as a function of surface for Experiment 2: (A) UHMW, (B) PVC, (C) glass, (D) rosin-coated glass, and (E) lubricated polyethylene. 

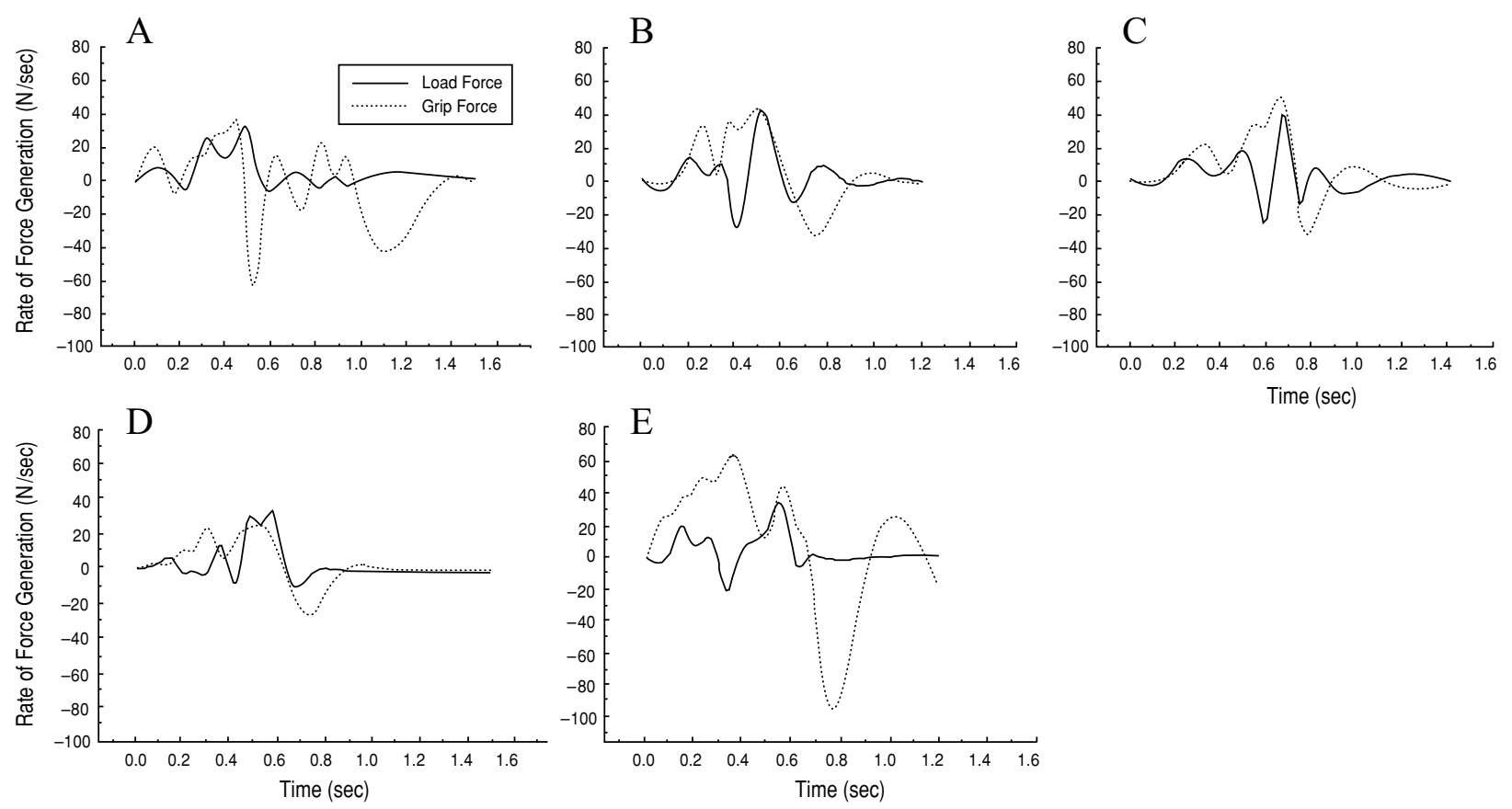

Figure 6. Typical rate of force generation profiles for 1 participant for each surface for Experiment 2: (A) UHMW, (B) PVC, (C) glass, (D) rosin-coated glass, and (E) lubricated UHMW.

force was larger than the force for Trials 3 through 10). The grip forces generated on the rosin-coated glass surface were higher than the grip forces generated on the three intermediate surfaces for Trials 2 and 4, with the lubricated polyethylene yielding the largest peak grip force on all trials (see Figure 7). These findings are contradictory to the findings of Cadoret and Smith (1996), who suggested that individu- als generate the least amount of grip force for surfaces with the highest coefficient of friction.

Major peak rate of grip force generation. The analysis of the rate of grip force generation (see Figures 6 and 8 ) showed a main effect of surface $[F(4,76)=28.67$, $p<.01]$. There was also a main effect of trial $[F(9,171)=$ $5.43, p<.01]$. The peak grip force generation rate for the

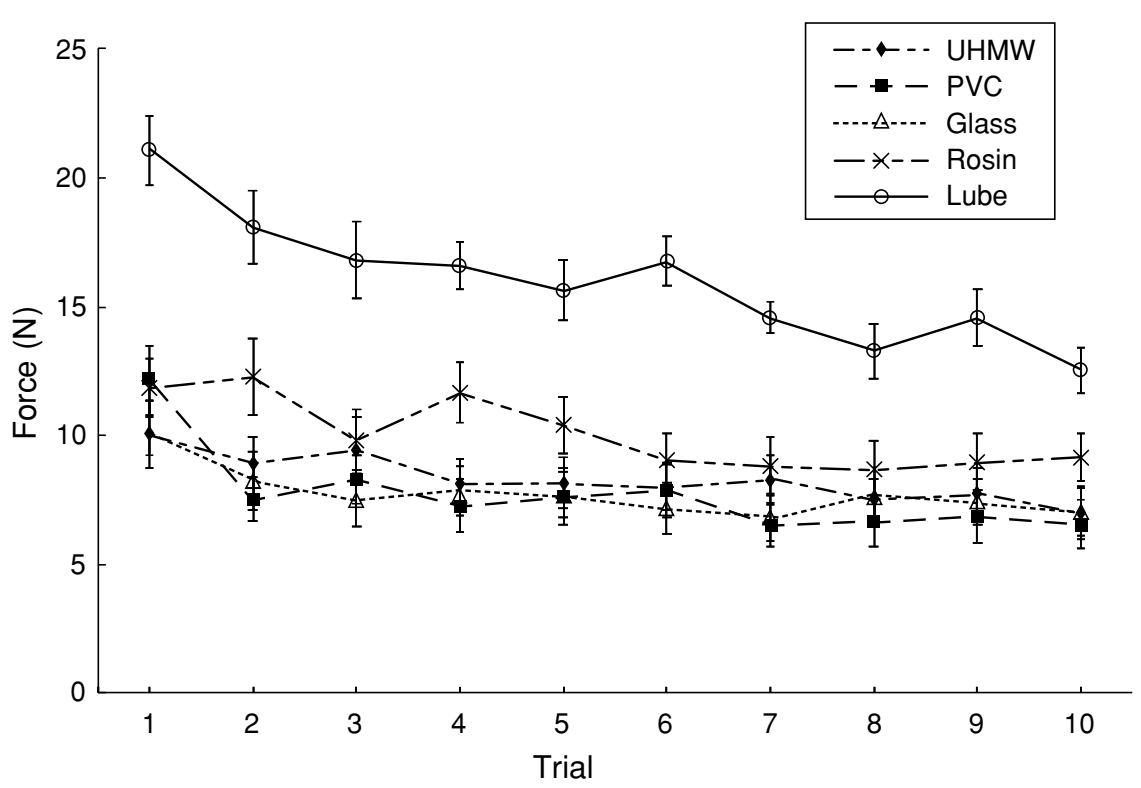

Figure 7. Peak grip force, plotted as a function of trial, for each surface in Experiment 2. 

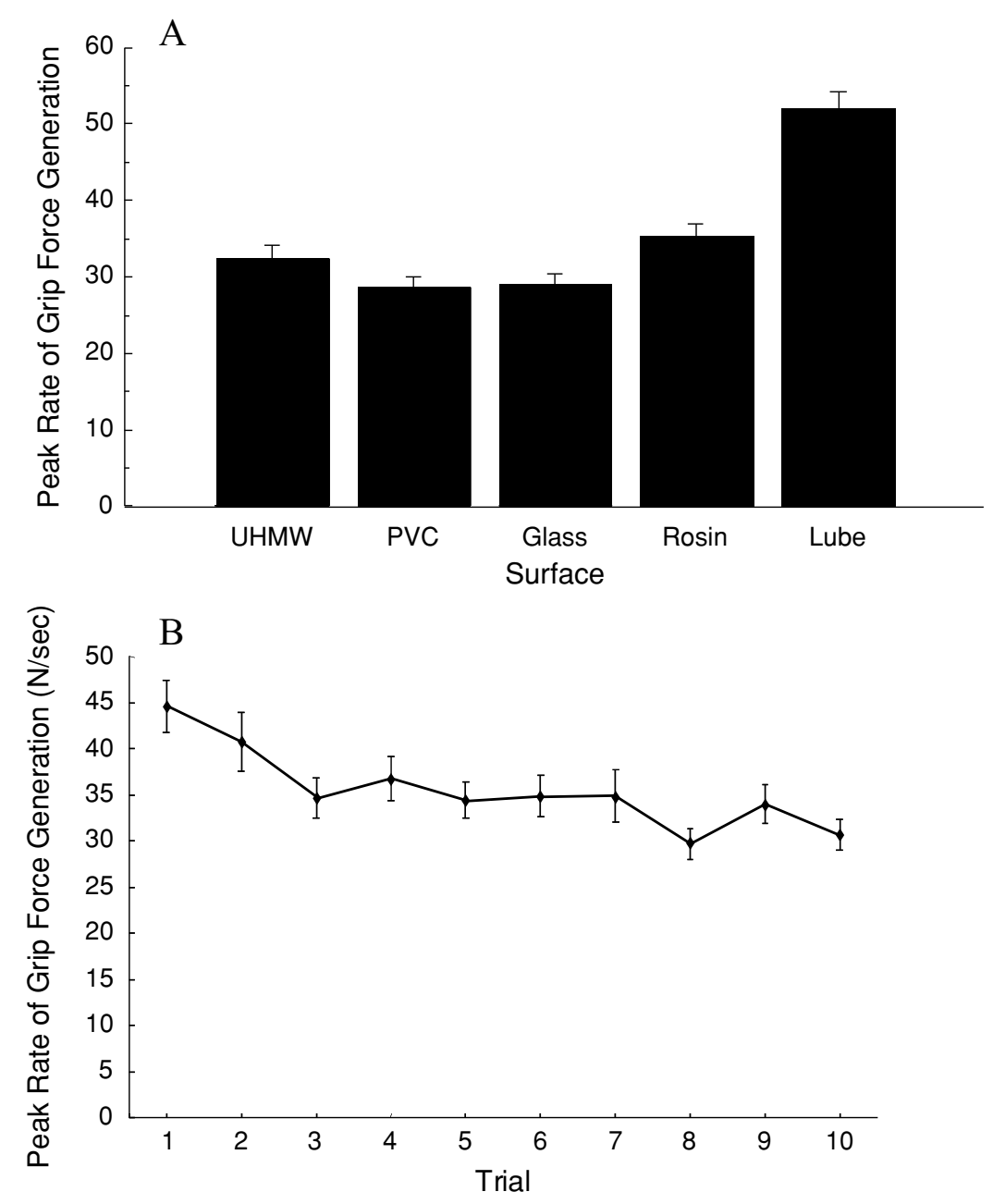

Figure 8. (A) Peak rate of grip force generation, plotted as a function of surface. (B) Peak rate of grip force generation, plotted as a function of trial.

lubricated polyethylene was significantly higher than the rates generated for the other four surfaces. This finding suggests that individuals must apply the grip force in a quicker fashion for the lubricated polyethylene in order to reach a coefficient of friction-suitable grip force by the time the object begins to lift off the table. The trial effect showed a general decrease in peak grip rate as a function of practice. More specifically, the first two trials had larger grip rates than did Trials 8, 9, and 10 .

Peak load force. The analysis of peak load forces showed no significant differences between any of the surfaces (see Figure 9A). This was to be expected, because load force should not be affected by changes in the coefficient of friction of the object being grasped (Cadoret \& Smith, 1996). There were also no statistically significant main effects or interaction with trial.

Major peak rate of load force generation. No differences were found in the analysis of the rate of load force generation (see Figure 9B).

Torque. There was a significant effect of surface when resultant torque was analyzed $[F(4,76)=83.45, p<.05]$.
There were no statistically significant main effects of trial and there was no surface $\times$ trial interaction. There were significant differences between all the surfaces except the PVC plastic and the glass (see Figure 10). It may also be possible that the blind nature of the procedure resulted in the participants making finger contact in decentralized locations on the grasping surfaces, such that the prehensile forces were not consistently through the grip axis defined by the joined centers of the two grasping surfaces. Such a variation in finger placements during grasp could be cause for the large differences in torque.

Grip force rate minor peaks: Frequency and scores. Each participant's grip force rate profile was examined for the presence of any minor peaks. Minor peaks in a rate profile are indicative of the participant's use of feedback to make corrections throughout the course of his or her lift. A single-peaked profile suggests that the forces incorporated were planned before the onset of the lift. No significant differences in the frequency of grip force rate minor peaks were found between the surfaces. There were minor peaks for $83 \%$ of all the trials. An ANOVA was also 

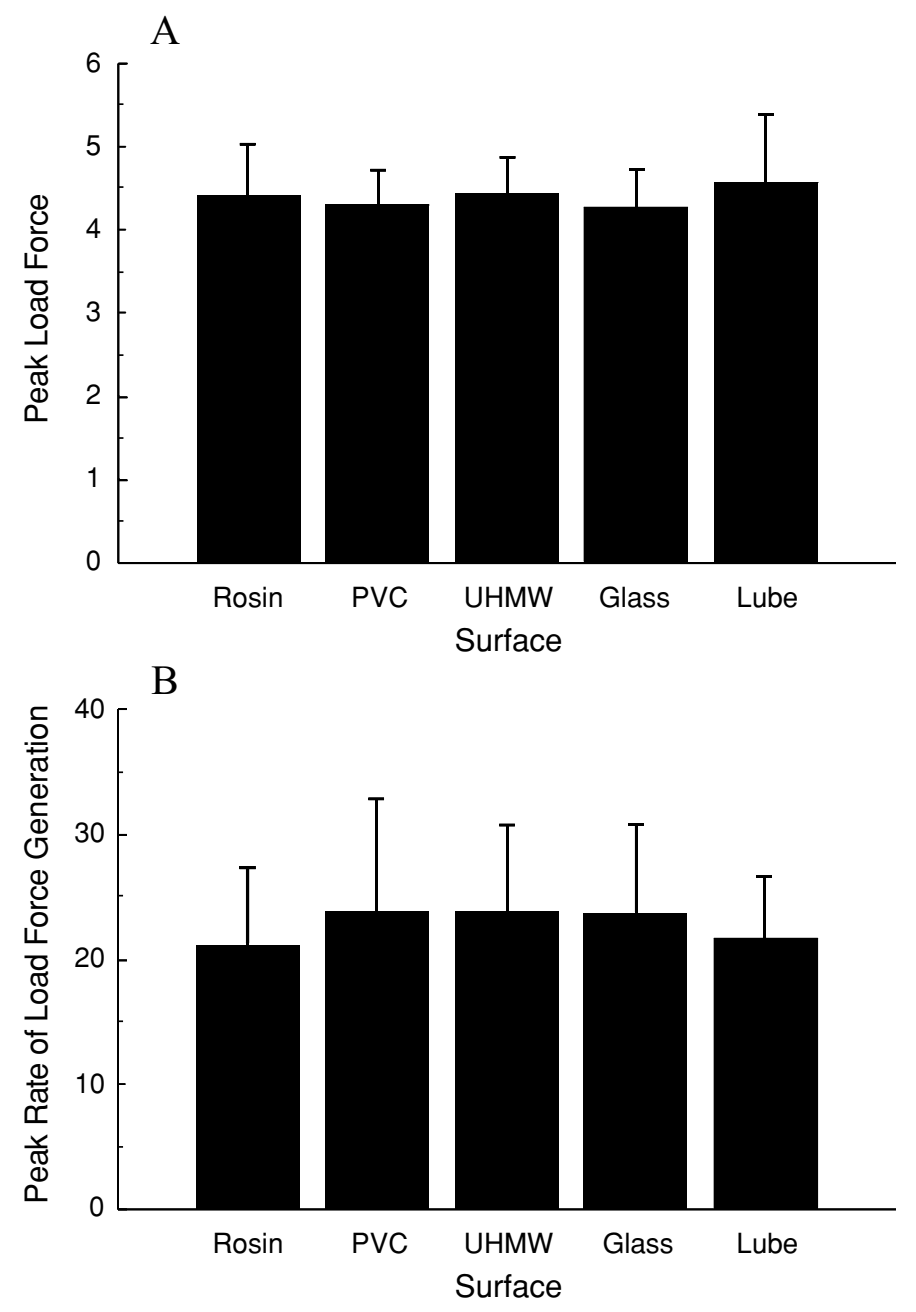

Figure 9. (A) Peak load force, plotted as a function of surface. (B) Peak rate of load force generation, as a function of surface.

conducted on the values of the grip force rate minor peaks. This analysis indicated a main effect of surface $[F(4,76)=$ $16.85, p<.05]$, with the lubricated polyethylene minor peaks being significantly larger than the minor peaks for the other four surfaces, and the rosin-coated glass minor peaks being significantly higher than the minor peaks for the PVC plastic (see Figures 11A and 11B).

Correlations. A significant correlation of $r=.297$ $(p<.01)$ was found between the perceptual estimates and the peak grip forces generated, indicating that the perceptual estimates increased as a function of the increases in peak grip force. This finding suggests a link between the generated grip force and the participants' ability to perceive skin-to-surface coefficient of friction. Additionally, the correlation between the participants' perceptual ratings and their peak rate of grip force generation was also significant $(r=.251, p<.01)$.

A significant inverse correlation $(r=-.305, p<.01)$ was found between the perceptual estimates and the skinto-surface coefficients of friction, indicating that the par- ticipants had some success in perceptually identifying the physical coefficient of friction of each surface during prehension. Contrary to the Experiment 1 finding that tangential motion is necessary for the perception of friction, this finding suggests that grasping with a static grip also allows for this perception. Perhaps there is enough tangential force caused by the mass of the object to stimulate tangentialforce-sensitive receptors (Smith \& Scott, 1996).

Interestingly, contrary to the findings of Cadoret and Smith (1996), no significant correlation was found between the peak grip force produced and the skin-tosurface coefficients of friction. The rosin-coated glass yielded a higher peak grip force, but was perceived as the least slippery surface. The correlation data were examined for each individual participant to address the possibility of any values disproportionately swaying the results. Thirteen participants showed significant correlations between peak grip force and the surfaces' coefficients of friction. The mean correlation coefficient of these 13 individuals was $r=-.467$. The remaining 7 participants produced 


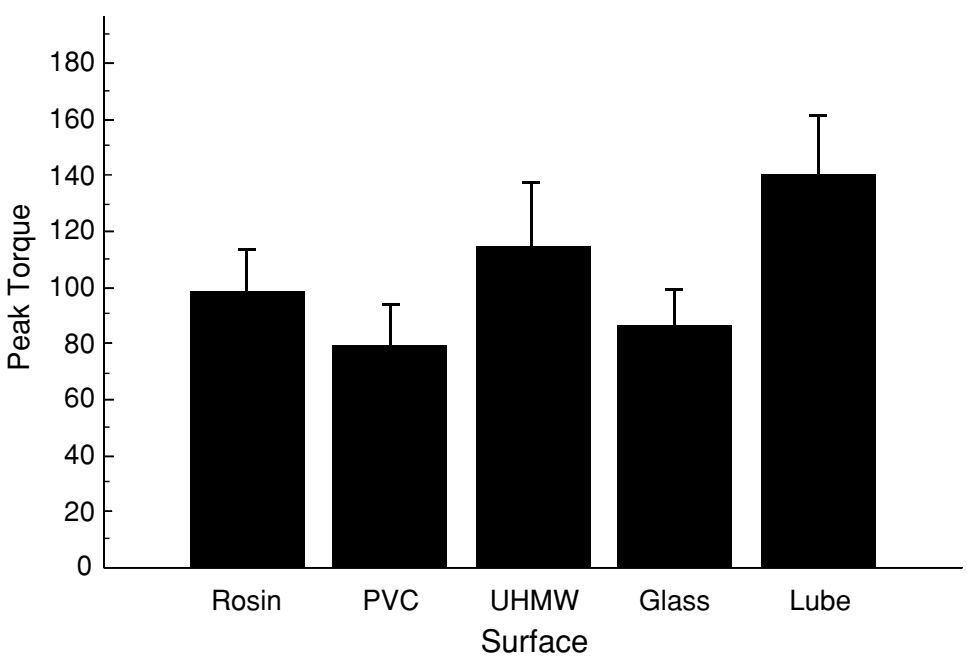

Figure 10. Peak torque, as a function of surface, for Experiment 2.

a nonsignificant correlation coefficient of $r=-.059$. Thirteen participants also showed significant correlation coefficients $(r=-.366)$ for the correlation between rate of grip force generation and the coefficients of friction.

The peak rate of grip force generation was also found to be significantly correlated to the torque produced $(r=$ $.129, p<.01)$. The generation of grip force at a greater rate may cause individuals to adopt less "stable" grasps, which could cause larger moments and, subsequently, larger torque.

In summary, the participants used online feedback to make corrections and adjustments during the lifts, as is evidenced by the majority of trials containing minor peaks for both the rates of grip force and load force generation. Thus, it can be stated that, to some degree, available haptic information was used by the participants to gauge their prehensile forces. Load forces and rate of load force generation were consistent with what is expected in prehen- sion when grasping surfaces' coefficients of friction are altered (Johansson \& Westling, 1984). Furthermore, differences in torque may be accounted for by the blind nature of the task. However, the generation of grip force was partially contradictory to the literature (Cadoret \& Smith, 1996; Johansson \& Westling, 1984). For the four surfaces with the lowest coefficients of friction there is no contradiction, but at the high end of the friction continuum, a dissociation existed. The rosin-coated glass, having the highest coefficient of friction, should have elicited the lowest peak grip force; but rather, a higher peak grip force than for three of the less slippery surfaces was generated. And since the participants were perceptually able to rate the slipperiness of the surfaces in accordance with the surfaces' actual coefficients of friction, there is evidence of a dissociation between the action and perception components of prehension in terms of identification of surface frictional quality.
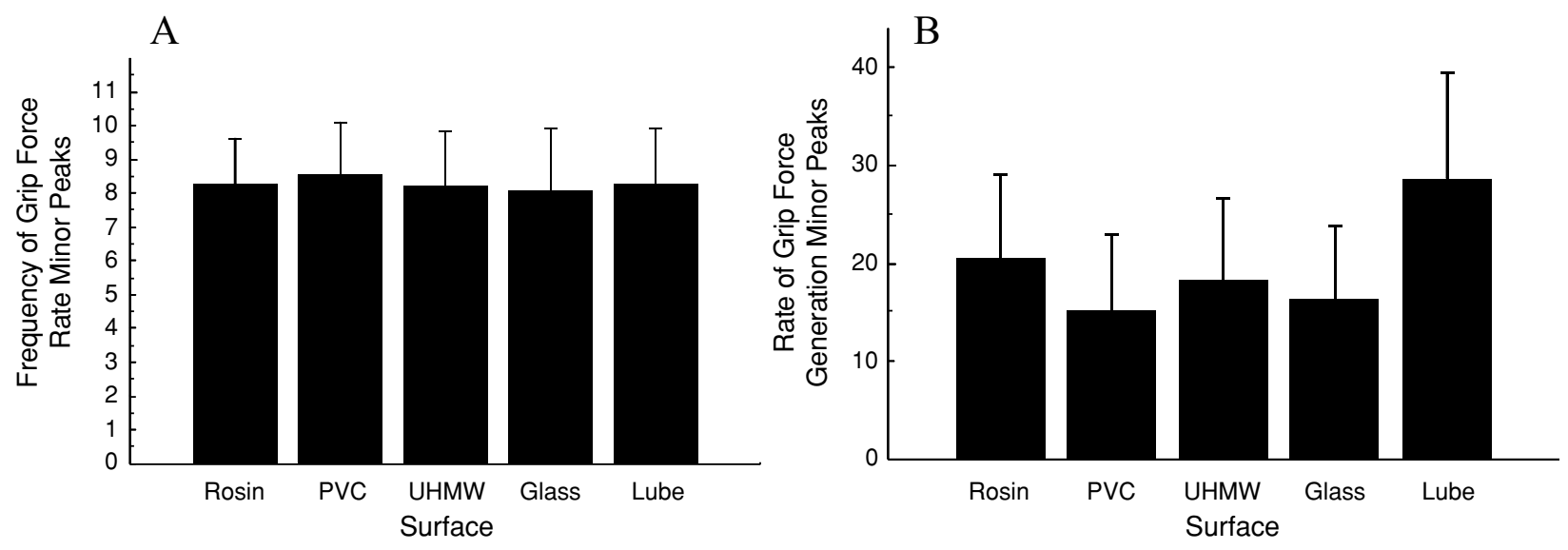

Figure 11. (A) Frequency of rate of grip force generation minor peaks, plotted as a function of surface. (B) Magnitude of rate of grip force generation minor peaks, plotted as a function of surface. 


\section{GENERAL DISCUSSION}

The results of Experiment 1 confirmed that accurate perceptions of surface friction could be formed only when a person generated tangential finger motion across the surface. Based on this, the initial hypothesis for the results of Experiment 2 was that during prehension, the slipperiness of the surface would be perceptually unavailable. Motorically, however, the participants were expected to generate force profiles that were appropriately scaled to the coefficient of friction that existed between the surface and the finger pad. Contrary to the initial hypotheses, the results showed that during prehension, individuals were able to perceive slipperiness that was significantly correlated to the actual skin-to-surface coefficients of friction, despite the lack of dynamic motion. Furthermore, the grip forces produced when grasping the various surfaces were scaled appropriately to the coefficients of friction for all surfaces except the rosin-coated glass surface. Not only was it a surprise that the results of the experiment were contrary to the initial hypothesis, such that the surface frictions were perceptually available, but it was also surprising that despite their accurate perceptions of friction, individuals produced uncharacteristically high grip forces when lifting the rosin-coated glass surface. It should be noted that the range of coefficients of friction in these studies was quite limited. In future work, it would be helpful to use a larger range that includes more intermediate steps in addressing the relationship between coefficient of friction, perception of slipperiness, and force control.

Johansson and Westling (1984) examined the roles of skin receptors in the control of precision grip when participants lifted slippery objects. The results of their work confirm that during prehension, the balance between the load and grip forces is adapted to the friction inherent between the skin and the surface to provide a small safety margin in order to prevent slips. As a result, for any given load force, higher grip forces are generated for slipperier surfaces. The results of applying a localized anesthesia to the finger pads showed that this adaptation is dependent on cutaneous afferent receptors. Johansson and Westling's findings have provoked many questions regarding the nature of these afferent inputs. Functionally, it is thought that the goal of haptic exploration is to optimize the dynamic nature of the exploration of a surface in order to recruit the maximal number of afferent receptors (Johnson, 1983). Srinivasan, Whitehouse, and LaMotte (1990) found that individuals were able to accurately perceive the direction of skin stretch through the use of slowly adapting mechanoreceptors, and that they appear to be more sensitive to textured surfaces with higher coefficients of friction. Although higher degrees of tangential force will evoke a larger response from most of the finger pads' slowly adapting mechanoreceptors, there seem to be some that respond to smoother textures with lower coefficients of friction (Smith, 1994).

Pare et al. (2002) conducted a study to assess whether individuals have the ability to generate magnitude estima- tions of varying levels of tangential force applied to the fingers. On a self-imposed magnitude-estimation scale, their participants showed that they were able to accurately judge the magnitudes of tangential force, but the rate of force application had no influence on their perceptions. The present study showed that, in the rolling condition, the participants were able to distinguish extreme coefficient of friction surfaces from the intermediate surfaces. Although they were able to perceive differences between the surfaces, the correlation data indicated that they were not able to make accurate judgments regarding the skinto-surface friction in this condition. It is entirely possible that the rolling condition does, in fact, because of its active component, provide a relevant stimulus to the mechanoreceptors of the finger. However, it is still unclear how the friction of the surfaces is differentiated during prehension. If the innervation of mechanoreceptors that respond to the magnitude and direction of shear force application allows for friction perception, how is it that friction can be perceived accurately for all the presented experimental conditions despite the unvarying magnitude and direction of the applied tangential force?

It has been suggested that finger slips across surfaces may provide information that maximizes the skin's extraction of afferent information and, in turn, allows conscious perceptions of skin-to-surface friction to be formed (Morley, Goodwin, \& Darian-Smith, 1983; Pare et al., 2002). These slips of the skin are considered to be perceptually unnoticeable, although they are revealed to the experimenters to be vibrations in the manipulated object (Johansson \& Westling, 1984). Johansson and Westling (1987), as well as Srinivasan et al. (1990), identified that the finger pads contain rapidly adapting cutaneous mechanoreceptors that respond to these slips, proposing that the best signal of slips occurs when smooth surfaces pass over these mechanoreceptors. It is suggested that during lifting and holding, these rapidly adapting mechanoreceptors provide a slip-related signal whose frequency is directly proportional to the surface friction, and conversely, whose signal duration is inversely proportional to both the surface roughness and the friction (Johansson \& Westling, 1987; Smith, 1994; Srinivasan et al., 1990). It has also been offered that these receptors may, indeed, be very adept at processing the information gained from slips. However, information gained from slips would be in opposition to the notion that grasp forces are applied in a manner intended to avoid slips (Pare et al., 2002). Indentation of the skin on simple contact with a surface may be sufficient to provide an estimation of surface friction without any further slip of the skin across the surface (Smith, 1994).

Although the research examining mechanoreceptors that are sensitive to microslips of the finger across a surface provides some possible answers about how surface friction is perceptually available during grasping, the question of why the rosin-coated glass elicited a larger than necessary grip force still needs to be addressed. A possibility offered here is that the haptic system of affer- 
ent information processing is functionally divided into an action component and a perception component, similar to the ventral and dorsal streams of the visual afferent information-processing system. The present study attempted to elicit a haptic action-perception dissociation through the manipulation of surface friction during prehension. Though a dissociation was seen, it was considerably weaker than action-perception disparities that are seen in vision. During the grasping portion of the second experiment, the participants demonstrated that they were perceptually proficient at consciously estimating the slipperiness of the surfaces they were lifting. From the results of Experiment 1, we can assume that some tangential motion, possibly identified by rapidly adapting slip receptors, occurred. Whether conscious or unconscious, the ability to perceive slipperiness is necessary in order to scale finger forces to a surface's frictional coefficient, so that the goal of performing a successful lift can be attained. On the whole, the motor outputs showed that the participants were able to do this for the majority of the presented surfaces except the least slippery one, the rosin-coated glass. For this surface, individuals produced higher grip forces and grip force rates, despite their accurate ability to rate the coefficient of friction of the surface, eliciting some evidence of an action-perception dissociation. Additional evidence of action-perception dissociation was seen in the response of the perceptual and motor systems to repeated trials. That is, whereas the perception of slipperiness increased for the PVC and glass surfaces as a function of trials, the force production decreased as a function of trials.

It is possible that the perception estimates that were produced after each lift may have affected their motor outputs. Milner and Goodale's (1995) model of the visual system proposes that the perceptual and motor streams are independent, suggesting that producing perceptual estimates should not influence motor output. However, the haptic system may not have the same independence between the perceptual and motor systems.

\section{REFERENCES}

Amazeen, E. L., \& Turvey, M. T. (1996). Weight perception and the haptic size-weight illusion are functions of the inertia tensor. Journal of Experimental Psychology: Human Perception \& Performance, 22, 213-232.

Blake, D. T., Hsiao, S. S., \& Johnson, K. O. (1997). Neural coding mechanisms in tactile pattern recognition: The relative contributions of slowly and rapidly adapting mechanoreceptors to perceived roughness. Journal of Neuroscience, 17, 7480-7489.

CAdoret, G., \& SMith, A. M. (1996). Friction, not texture, dictates grip forces used during object manipulation. Journal of Neurophysiology, 75, 1963-1969.

Charpentier, A. (1891). Analyse expérimentale de quelques éléments de la sensation de poids [Experimental study of some aspects of weight perception]. Archives de Physiologie Normale et Pathologique, 3, 122-135.
Ellis, R. R., \& Lederman, S. J. (1993). The role of haptic versus visual volume cues in the size-weight illusion. Perception \& Psychophysics, 53, 315-324.

Gordon, A. M., Forssberg, H., Johansson, R. S., \& Westling, G. (1991a). Visual size cues in the programming of manipulative forces during precision grip. Experimental Brain Research, 83, 477-482.

Gordon, A. M., Forssberg, H., Johansson, R. S., \& Westling, G. (1991b). The integration of haptically acquired size information in the programming of precision grip. Experimental Brain Research, 83, 483-488.

James, T. W., Humphrey, G. K., Gati, J. S., Servos, P., Menon, R. S., \& Goodale, M. A. (2002). Haptic study of three-dimensional objects activates extrastriate visual areas. Neuropsychologia, 40, 1706-1714.

Johansson, R. S., \& Westling, G. (1984). Roles of glabrous skin receptors and sensorimotor memory in automatic control of precision grip when lifting rougher or more slippery objects. Experimental Brain Research, 56, 550-564.

Johansson, R. S., \& Westling, G. (1987). Signals in tactile afferents from the fingers eliciting adaptive motor responses during precision grip. Experimental Brain Research, 66, 141-154.

Johansson, R. S., \& Westling, G. (1990). Tactile afferent signals in the control of precision grip. In M. Jeannerod (Ed.), Attention and performance XIII: Motor representation and control (pp. 677-713). Hillsdale, NJ: Erlbaum.

Johnson, K. O. (1983). Neural mechanisms of tactual form and texture discrimination. Federation Proceedings, 42, 2542-2547.

JoHnson, K. O. (2001). The roles and functions of cutaneous mechanoreceptors. Current Opinion in Neurobiology, 11, 455-461.

Johnson, K. O., Hsiao, S. S., \& Yoshioka, T. (2002). Neural coding and the basic law of psychophysics. Neuroscientist, 8, 111-121.

Lederman, S. J., \& TAYlor, M. M. (1972). Fingertip force, surface geometry, and the perception of roughness by active touch. Perception \& Psychophysics, 12, 401-408.

Milner, D. A., \& Goodale, M. A. (1995). The visual brain in action. Oxford: Oxford University Press.

Morley, J. W., Goodwin, A. W., \& Darian-Smith, I. (1983). Tactile discrimination of gratings. Experimental Brain Research, 49, 291-299.

Pare, M., Carnahan, H., \& Smith, A. M. (2002). Magnitude estimation of tangential force applied to the fingerpad. Experimental Brain Research, 142, 342-348.

Prather, S. C., Votaw, J. R., \& Sathian, K. (2004). Task-specific recruitment of dorsal and ventral visual areas during tactile perception. Neuropsychologia, 42, 1079-1087.

Shockley, K., Carello, C., \& Turvey, M. T. (2004). Metamers in the haptic perception of heaviness and moveableness. Perception \& Psychophysics, 66, 731-742.

Smith, A. M. (1994). Some shear facts and pure friction related to roughness discrimination and the cutaneous control of grasping. Canadian Journal of Physiology \& Pharmacology, 72, 583-590.

Smith, A. M., \& ScotT, S. H. (1996). Subjective scaling of smooth surface friction. Journal of Neurophysiology, 75, 1957-1962.

Srinivasan, M. A., Whitehouse, J. M., \& LaMotte, R. H. (1990). Tactile detection of slip: Surface microgeometry and peripheral neural codes. Journal of Neurophysiology, 63, 1323-1332.

Symmons, M., \& Richardson, B. (2000). Raised line drawings are spontaneously explored with a single finger. Perception, 29, 621-626.

Westwood, D. A., \& Goodale, M. A. (2003). A haptic size-contrast illusion affects perception but not grasping. Experimental Brain Research, 153, 253-259.

(Manuscript received July 8, 2004 revision accepted for publication October 11, 2005.) 